\title{
Genetic Structure and Drug Susceptibility Patterns of Mycobacterium tuberculosis Complex Strains Responsible of Human Pulmonary Tuberculosis in the Major Rearing Region in Cameroon
}

\author{
Francioli Koro Koro, ${ }^{1,2}$ Alphonse Um Boock, ${ }^{3}$ Afi Leslie Kaiyven, ${ }^{1}$ Juergen Noeske, \\ Cristina Gutierrez, ${ }^{5}$ Christopher Kuaban, $^{6}$ François-Xavier Etoa, ${ }^{7}$ and Sara Irène Eyangoh ${ }^{1}$ \\ ${ }^{1}$ Mycobacteriology Service, Reference Laboratory of NTP, Centre Pasteur of Cameroon, \\ Cameroon-Pasteur Institute International Network, Yaoundé, Cameroon \\ ${ }^{2}$ Department of Biochemistry, Faculty of Sciences, University of Douala, Yaoundé, Cameroon \\ ${ }^{3}$ FAIRMED Foundation, Yaoundé, Cameroon \\ ${ }^{4}$ German International Cooperation (GIZ), Yaoundé, Cameroon \\ ${ }^{5}$ Caribbean Public Health Agency, Port of Spain, Trinidad and Tobago \\ ${ }^{6}$ Faculty of Medicine and Biomedical Sciences/Jamot Hospital, University of Bamenda, Yaoundé, Cameroon \\ ${ }^{7}$ Department of Microbiology, Faculty of Sciences, University of Yaoundé I, Yaoundé, Cameroon
}

Correspondence should be addressed to Francioli Koro Koro; korokorogozion@yahoo.fr

Received 24 August 2016; Revised 15 November 2016; Accepted 7 December 2016

Academic Editor: Gernot Zissel

Copyright (C) 2016 Francioli Koro Koro et al. This is an open access article distributed under the Creative Commons Attribution License, which permits unrestricted use, distribution, and reproduction in any medium, provided the original work is properly cited.

\begin{abstract}
Background. Cameroon this last decade continues to present a low contribution of M. africanum and M. bovis in human tuberculosis (TB), while M. bovis was prevalent in cattle but all these pieces of information only concerned West and Center regions. Methods. We carried out the first study in Adamaoua, one of the most rearing regions of Cameroon, on the genetic structure and drug susceptibility of the MTBC strains isolated from newly diagnosed sputum smear-positive patients aged 15 years and above. For that purpose, spoligotyping, a modified 15 standard MIRU/VNTR loci typing, and the proportion method were used. Results. Four hundred and thirty-seven MTBC isolates were analyzed by spoligotyping. Of these, 423 were identified as M. tuberculosis, within the Cameroon family being dominant with 278 (65.7\%) isolates; twelve $(2.75 \%)$ isolates were classified as M. africanum and two as M. bovis. MIRU/VNTR typing of the most prevalent sublineage (SIT 61) suggested that this lineage is not a unique clone as thought earlier but could constitute a group of strains implicated to different pocket of TB transmission. Only M. tuberculosis sublineages were associated with antituberculosis drug resistance. Conclusion. These results showed the weak contribution of M. africanum and M. bovis to human active pulmonary tuberculosis in Cameroon even in the rearing region.
\end{abstract}

\section{Introduction}

Cameroon is an intermediary country with regard to the incidence of tuberculosis (TB) in sub-Saharan Africa, which is estimated at $122 \mathrm{~TB}$ cases per 100,000 per year [1]. Cameroon is also situated in the Central West African region, where there is a particular dominance of a closely related group of Mycobacterium tuberculosis strains, called Cameroon family and the decline of $M$. africanum in human TB $[2,3]$. The reasons of this successful adaptation of $M$. tuberculosis, the genuine regression of M. africanum, and the constant absence of Beijing strains, need to be explored for a better understanding of the major driving forces of the dynamics in transmission within specific populations. This may significantly impact TB control and vaccine strategies development.

It is known that molecular typing of M. tuberculosis complex strains can greatly enhance our understanding of population structure and strains circulation of the MTBC and may 
help improve TB control [4], especially in Cameroun where there seems to be a rapid dynamic evolution or change in the $M$. tuberculosis complex strain population structure since 1970.

In fact, in Cameroon, the application of spoligotyping used in sparse studies showed a striking regression of $M$. africanum as etiologic agent of pulmonary tuberculosis in the West and Center regions $[2,3,5]$. These studies also showed the predominance of a group of $M$. tuberculosis strains named "Cameroon family" $[2,3]$. This can be intriguing considering the population structure or the prevalence of $M$. africanum strain in Nigeria, a neighbouring country to Cameroon, and in other West African countries.

Adamaoua is a breeding region of Cameroon with an approximate population of 703,432 and with an estimated incidence of $121 \mathrm{~TB}$ cases per 100,000 per year [1]. It is also neighboring Nigeria and Central African Republic, two high TB burden countries in Africa [6].

Nowadays, there is very limited data available pertaining strains circulating and the population structure of $M$. tuberculosis in other regions of Cameroon apart from the West and Center regions.

The aims of this study were to evaluate for the first time the genetic population structure of $M$. tuberculosis complex strains in Adamaoua and to estimate the implication of $M$. bovis in human pulmonary tuberculosis, in order to gain better understanding of the population structure of MTBC and strains circulation in Cameroon.

\section{Methods}

2.1. Ethical Considerations. Institutional permission, reference number $052 / \mathrm{CNE} / \mathrm{DNM} / 07$, to conduct the study was obtained from the National Ethics Committee of the Public Health Ministry. The patients were included in the study after understanding the aims of the research and having signed an informed consent.

2.2. Study Design. This was a cross-sectional study which included all consecutive newly presenting Ziehl-Neelsen (ZN) smear-positive patients between June 2008 and December 2009, aged 15 years and above. The patient recruitment and sample collection process was done as described in our previous report $[2,3]$. Briefly, all patients underwent a standardized interview and three consecutive sputum samples were collected from each. Only the samples with positive $\mathrm{ZN}$ smear were further analyzed. Samples were stored in cetyl bromide pyrinidium $0.6 \%(\mathrm{~V} / \mathrm{V})$ at the recruitment DCT clinics and transported according to International Union Against Tuberculosis and Lung Diseases recommendations [7] by train to the NTRL for processing, confirmatory ZiehlNeelsen microscopy, and culture and molecular analysis.

2.3. Sputum Sample Processing. Specimens (2-5 mL) were washed three times with sterile distilled water in proportion $1: 3(\mathrm{~V} / \mathrm{V})$ and concentrated each time at $4000 \times \mathrm{rpm}$ for 20 minutes. The sediment, irrespective of the original sample volume, was reconstituted to three-milliliter sterile distilled water to make the inoculums for the smears and cultures.

2.4. Culture and Identification. Three Lowenstein-Jensen (LJ) slants, two containing $0.75 \%$ glycerol without pyruvate and one containing $0.4 \%$ pyruvate, were inoculated with the sediment and incubated at $37^{\circ} \mathrm{C}$. Cultures were considered negative when no colonies were seen after eight weeks of incubation. Isolates were harvested and DNA extraction was performed as described in our previous report [2,3].

The drug susceptibility pattern of all identified mycobacterial isolates to isoniazid $(0.2 \mathrm{mg} / \mathrm{mL}$ and $1 \mathrm{mg} / \mathrm{mL})$, rifampicin $(40 \mathrm{mg} / \mathrm{mL})$, streptomycin $(4 \mathrm{mg} / \mathrm{mL})$, and ethambutol $(2 \mathrm{mg} / \mathrm{mL})$ was determined phenotypically by the indirect proportion method on L-J slants, as described previously [8]. Drug resistance was expressed as the proportion of colonies that grow on drug containing medium to drug-free medium and the critical proportion for resistance was $1 \%$ for all drugs.

2.5. Spoligotyping. Standard spoligotyping was done generally as described by Kamerbeek and colleagues [9] using a commercially available kit (Isogen Bioscience BV, Maarssen, The Netherlands).

2.6. MIRU/VNTR Typing. A modified standard 15-MIRU/ VNTR locus system MIRU04, ETRC, QUB-26, QUB-11b, MIRU24, MIRU20, MIRU40, ETRA, MIRU27, MIRU26, MIRU31, MIRU39, Mtub30, Mtub34, and Mtub21 were individually amplified and analyzed as previously described [10]. This system was chosen because it has a more reliable discrimination power between MTBC species, lineage, and clad when we analyze MTBC strains isolated from human and animal in our laboratory (personal data) compared to the conventional 12 and 15 loci that had been used before and gave the same haplotype for different species, lineages, or clad. Moreover this system permits the recovery of another polymorphic locus compared to that given by 12 and 15 standard system when we analyze Cameroonian strains. Results from each of the 15 loci were combined to form a 15-digit allele profile.

2.7. Data Analysis. The rate of diversity was calculated by dividing the number of spoligotypes by the number of isolates. The Hunter-Gaston discriminatory index (HGDI) was used to estimate the discriminatory power of spoligotyping and MIRU VNTR [11]. Fisher's exact and chi square test were used to estimate the association between resistance profile and genotype or sex or age or to evaluate the repartition of genotypes between Adamaoua and West region, using statistical software R version 2.15.3 (https://www.r-project.org/). Two-sided $p$ values of 0.05 or lower were considered statistically significant.

2.8. Family Assignment. The spoligotypes patterns were introduced to the SITVIT_WEB database (http://www.pasteurguadeloupe.fr:8081/SITVIT_ONLINE/) and assigned a SIT number. Major spoligotyping-based phylogeographic clades 
TABLE 1: Antituberculosis drug resistance profiles in Adamaoua region.

\begin{tabular}{|c|c|c|c|c|}
\hline Resistance to & Antituberculosis drugs & $\begin{array}{l}\text { Total number of } \\
\text { resistant strains }\end{array}$ & $\begin{array}{c}\% \text { according to all } \\
\text { new cases }(n=437)\end{array}$ & $\begin{array}{l}\text { \% according to resistant cases } \\
(n=38)\end{array}$ \\
\hline \multirow{4}{*}{ One drug } & $\mathrm{H}$ & 13 & 2,97 & 34,21 \\
\hline & $\mathrm{R}$ & 2 & 0,46 & 5,26 \\
\hline & S & 12 & 2,74 & 31,6 \\
\hline & Total & 27 & 6,99 & 71,05 \\
\hline \multirow{3}{*}{ Two drugs } & HR & 2 & 0,46 & 5,3 \\
\hline & HS & 3 & 0,68 & 7,9 \\
\hline & Total & 5 & 1.10 & 13,16 \\
\hline \multirow{3}{*}{ Three drugs } & HRS & 1 & 0,23 & 2,63 \\
\hline & RSE & 2 & 0,46 & 2,63 \\
\hline & Total & 3 & 0,68 & 5,3 \\
\hline Four drugs & HRSE & 3 & 0,68 & 7,9 \\
\hline Total resistance & & 38 & 8,70 & 100 \\
\hline \multirow[t]{2}{*}{ Total sensitive strains } & & 399 & 91,30 & \\
\hline & Total & 437 & 100 & \\
\hline
\end{tabular}

were assigned according to signatures provided by Brudey and collaborators [12].

\section{Results}

3.1. Studied Patients Data. This was a cross-sectional study of 452 newly diagnosed TB patients aged between 15 and 85 years with mean age of 32.4 years. The female to male sex ratio was approximately $2: 3$. The TB-HIV coinfection rate was $26.55 \%$ (94/354) of which $54.3 \%$ (51/94) were women and $45.8 \%(43 / 94)$ were men.

3.2. Drug Susceptibility Result. The primary resistance rate of isolates was $7.20 \%$ while primary resistance to one, two, three, or four antituberculosis drugs was observed in $6.99 \%, 1.1 \%, 0.67 \%$, and $0.67 \%$, respectively (Table 1 ). The primary monoresistance to isoniazid and streptomycin was the most prevalent (2.58\% both) followed by monoresistance to rifampicin $(0.52 \%)$. No monoresistance was recorded for ethambutol. Multidrug-resistance rate to at least isoniazid and rifampicin was $0.77 \%$. There was no statistical association between resistance, age, and sex (Table 1).

3.3. Spoligotype Results. Molecular typing of the 437 recovered strains by spoligotyping revealed that 423 strains belonged to $M$. tuberculosis species, 12 to $M$. africanum species, and two to $M$. bovis species.

The twelve M. africanum strains were regrouped into nine distinct spoligotypes patterns (Table 2) among which eight patterns were not clustered and one pattern was clustered into four strains, while the two $M$. bovis strains revealed two distinct spoligotypes patterns (Table 2).

Spoligotyping of the $423 \mathrm{M}$. tuberculosis isolates revealed 54 distinct spoligotypes, among which 29 were not clustered and the remaining 25 patterns were clustered into 2 and 217 strains.
The comparison between the spoligotypes found in our study and those of the SITVIT_WEB database revealed that only 41 had a SIT number. The remaining 24 were orphan within which 20 were not clustered and four clustered with 2-3 strains.

Among the $M$. africanum spoligotype patterns, only four were already described in SITVIT2. The other five were not clustered and had no SIT number. AFRI-2 lineage accounted for eight strains among which four were clustered (SIT 101) while AFRI-3 accounted for four strains representing four spoligotypes not clustered (Table 2).

The two M. bovis spoligotypes belonged to BOVIS1 (SIT 1037) and BOV (SIT 308) lineage.

Of the $423 \mathrm{M}$. tuberculosis strains, a total of $278(65.7 \%)$ belonged to the Cameroon family, of which 223 (80.2\%) strains were from the most prevalent clade called SIT61. The other described $M$. tuberculosis families were the ubiquitous $\mathrm{T}$ (69 strains), represented by lineage T1 (42 strains) and T2 (27 strains), the Haarlem (40 strains), represented by lineage $\mathrm{H} 1$ (20 strains) and H3 (20 strains), the U family represented by lineage $U$ (16 strains) and $U$ (Likely $H$ ) (13 strains), the CAS family represented by CAS1_Delhi (one strain), and the $\mathrm{X}$ family represented only by lineage X1 (one strain) which has never been described before in Cameroon. None of the strains had the spoligotype of the worldwide-expanded epidemic Beijing family (Table 2).

3.4. Drug Susceptibility Results among Mycobacterium tuberculosis Complex Species and Lineages. Regarding cluster analysis in drug resistant isolates, none of the M. africanum and $M$. bovis strains was responsible of drug resistance, while all drug resistance observed was exclusively due to $M$. tuberculosis strains. The $\mathrm{U}, \mathrm{T}$, and Cameroon family and the $\mathrm{H}$ families were principally responsible for $10.71 \%, 10.14 \%, 8.63 \%$, and $2.50 \%$ of all forms of antituberculosis drug resistance in Cameroon (Table 3). When looking for the MDR cases, the 
TABLE 2: Families and lineage among the 437 M. tuberculosis complex strains isolated from TPM+ patients from Adamaoua, Cameroon.

\begin{tabular}{|c|c|c|c|c|c|}
\hline Genetic families & Lineages & $\begin{array}{l}\text { Total number of } \\
\text { spoligotype }\end{array}$ & $\begin{array}{c}\text { Total number of } \\
\text { strains }\end{array}$ & $\begin{array}{l}\% \text { according to } \\
\text { total number of } \\
\text { strains }\end{array}$ & $\begin{array}{l}\% \text { according to } \\
\text { total number of } \\
\text { strains in the } \\
\text { family }\end{array}$ \\
\hline LAM & LAM1 & 1 & 4 & 0.91 & 100 \\
\hline Cameroon & LAM10_CAM & 9 & 278 & 63.61 & 100 \\
\hline \multirow{3}{*}{$\mathrm{T}$} & $\mathrm{T} 1$ & 7 & 42 & 9.61 & 60.9 \\
\hline & $\mathrm{T} 2$ & 12 & 27 & 6.18 & 39.1 \\
\hline & ND & 1 & 1 & 0.22 & - \\
\hline \multirow{2}{*}{ Haarlem } & $\mathrm{H} 1$ & 8 & 20 & 4.57 & 50.0 \\
\hline & H3 & 5 & 20 & 4.57 & 50.0 \\
\hline \multirow{2}{*}{$\mathrm{U}$} & $\mathrm{U}$ & 8 & 16 & 3.66 & 55.2 \\
\hline & U (likeli H) & 1 & 13 & 2.97 & 44.8 \\
\hline \multirow{2}{*}{ Africanum } & AFRI 2 & 5 & 8 & 1.83 & 66.7 \\
\hline & AFRI 3 & 4 & 4 & 0.91 & 33.3 \\
\hline \multirow{2}{*}{ Bovis } & BOV & 1 & 1 & 0.22 & 50 \\
\hline & BOVIS 1 & 1 & 1 & 0.22 & 50 \\
\hline CAS & CAS1_Deli & 1 & 1 & 0.22 & 100 \\
\hline $\mathrm{X}$ & $\mathrm{X} 1$ & 1 & 1 & 0.22 & 100 \\
\hline Total & & 65 & 437 & 100 & \\
\hline
\end{tabular}

$\mathrm{ND}=$ not determined

TABLE 3: Mycobacterium tuberculosis complex species and lineage drug susceptibility.

\begin{tabular}{|c|c|c|c|c|c|c|}
\hline \multirow[t]{2}{*}{ Family or lineage } & \multirow[t]{2}{*}{ Total strains } & \multirow{2}{*}{ Total primary resistance } & \multicolumn{3}{|c|}{$\begin{array}{c}\text { Percentage of } \\
\text { Primary monoresistance }\end{array}$} & \multirow{2}{*}{ MDR tuberculosis } \\
\hline & & & INH & RIF & STR & \\
\hline M. tuberculosis & 423 & $8.27(35 / 423)$ & $3.07(13 / 423)$ & $0.47(2 / 423)$ & $2.84(423)$ & $1.42(6 / 423)$ \\
\hline Cameroon family & 278 & $8.63(24 / 278)$ & $3.95(11 / 278)$ & $\mathbf{0 . 3 6}(1 / 278)$ & $3.24(9 / 278)$ & $0.36(1 / 278)$ \\
\hline $\mathrm{T} 1$ & 42 & $14.29(6 / 42)$ & $4.76(2 / 42)$ & 0 & $4.76(2 / 42)$ & $7.14(3 / 42)$ \\
\hline $\mathrm{T} 2$ & 27 & $3.70(1 / 27)$ & 0 & 0 & $3.70(1 / 27)$ & 0 \\
\hline $\mathrm{U}$ & 16 & $6.30(1 / 16)$ & 0 & 0 & 0 & $6.30(1 / 16)$ \\
\hline U (likely H) & 13 & $15.38(2 / 13)$ & 0 & $7.7(1 / 13)$ & 0 & $7.70(1 / 13)$ \\
\hline $\mathrm{H} 3$ & 20 & $5.00(1 / 20)$ & 0 & 0 & 0 & 0 \\
\hline $\mathrm{H} 1$ & 20 & 0 & 0 & 0 & 0 & 0 \\
\hline $\mathrm{X} 1$ & 1 & 0 & 0 & 0 & 0 & 0 \\
\hline CAS1_Delhi & 1 & 0 & 0 & 0 & 0 & 0 \\
\hline LAM1 & 4 & 0 & 0 & 0 & 0 & 0 \\
\hline M. africanum & 12 & 0 & 0 & 0 & 0 & 0 \\
\hline M. bovis & 2 & $\mathbf{0}$ & $\mathbf{0}$ & $\mathbf{0}$ & $\mathbf{0}$ & $\mathbf{0}$ \\
\hline Total & 436 & & & & & \\
\hline
\end{tabular}

Cameroon family, the $\mathrm{U}$ lineage, the $\mathrm{U}$ (likely $\mathrm{H}$ ) lineage, and the $\mathrm{T} 1$ lineage were responsible for $0.36 \%, 6.30 \%, 7.10 \%$, and $7.70 \%$ of the MDR case, respectively (Table 3 ). There was no statistical association between resistance and MTC genotypic families $(p>0.05)$.

3.5. Comparison of Genetic Population Structures of MTC Strains between Adamaoua and West Region in Cameroon. In order to understand strains circulation in Cameroon, we compared these data to those of our previous studies in the
West region of Cameroon. We found that twenty-nine (44.6\%) spoligotypes among the sixty-five found in Adamaoua were also found in West region. This represents 395 (90.4\%) of all isolates. From this, clades SIT61 and SIT53 were more distributed (Table 4) in the two regions. This result shows a common sharing of strains between these two regions of Cameroon.

On the other hand, strains of some lineages seemed to be more adapted in one of the two regions. For example, 73 strains of SIT 50 belonging to $\mathrm{H} 3$ lineage were isolated in West 


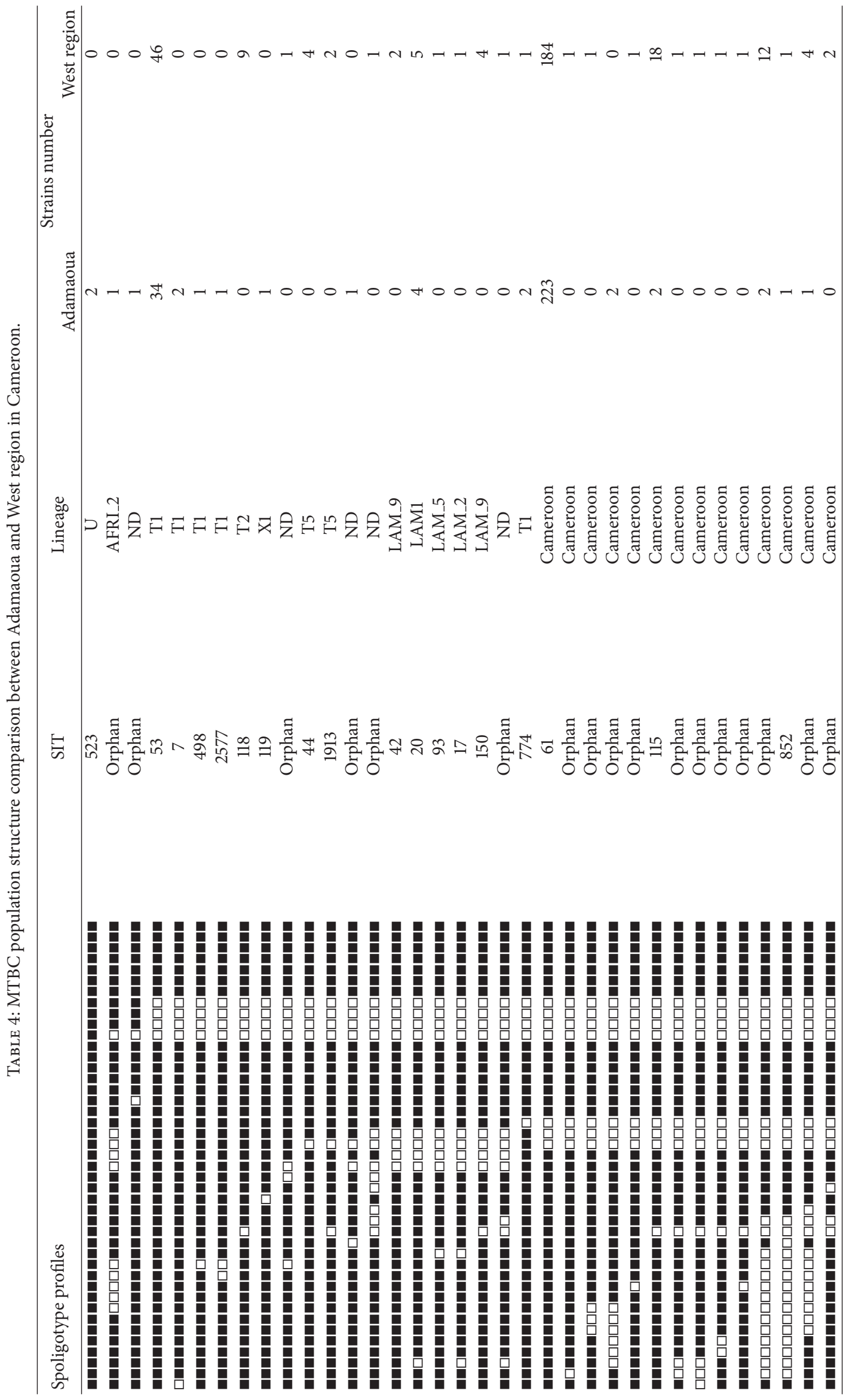




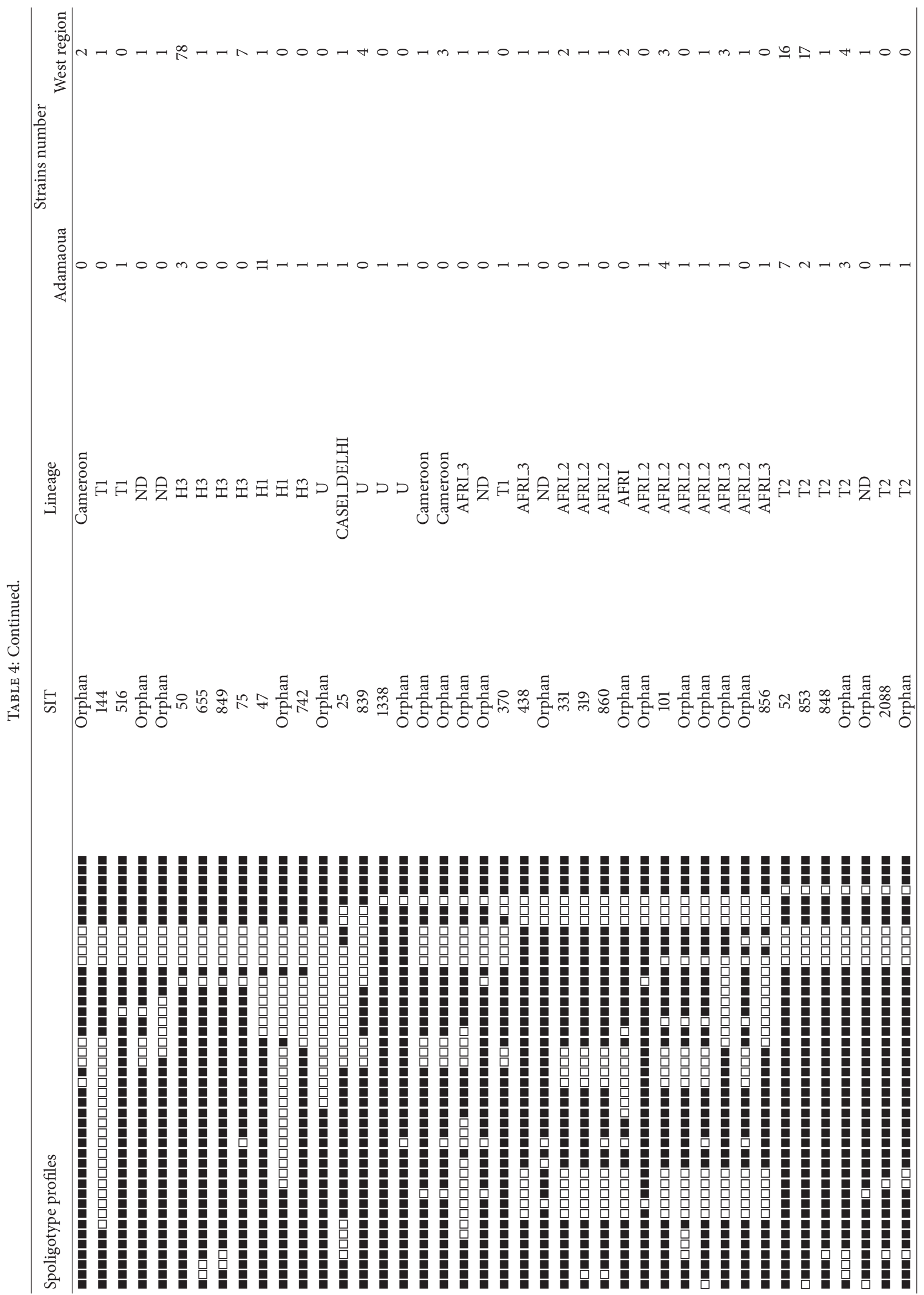




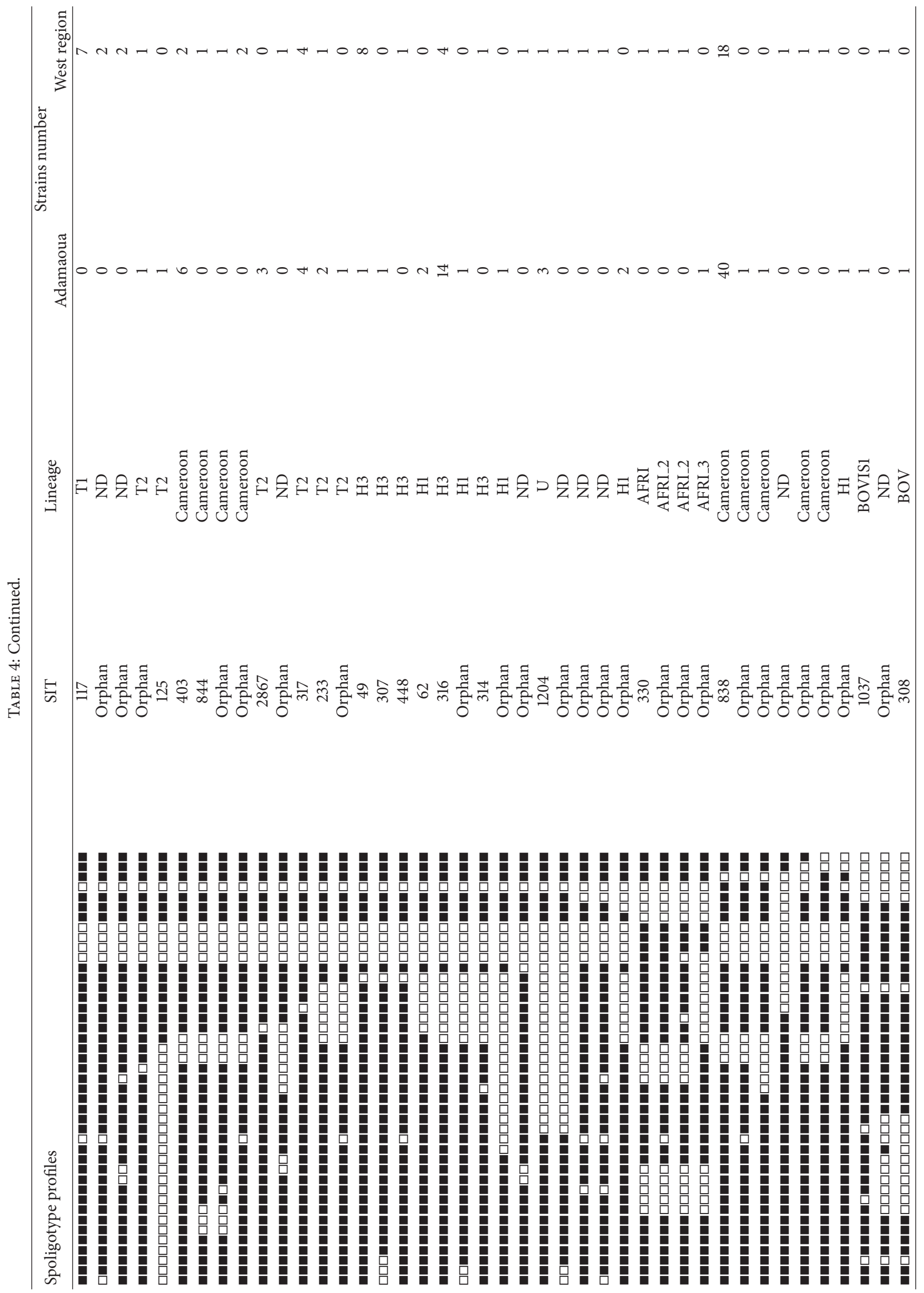




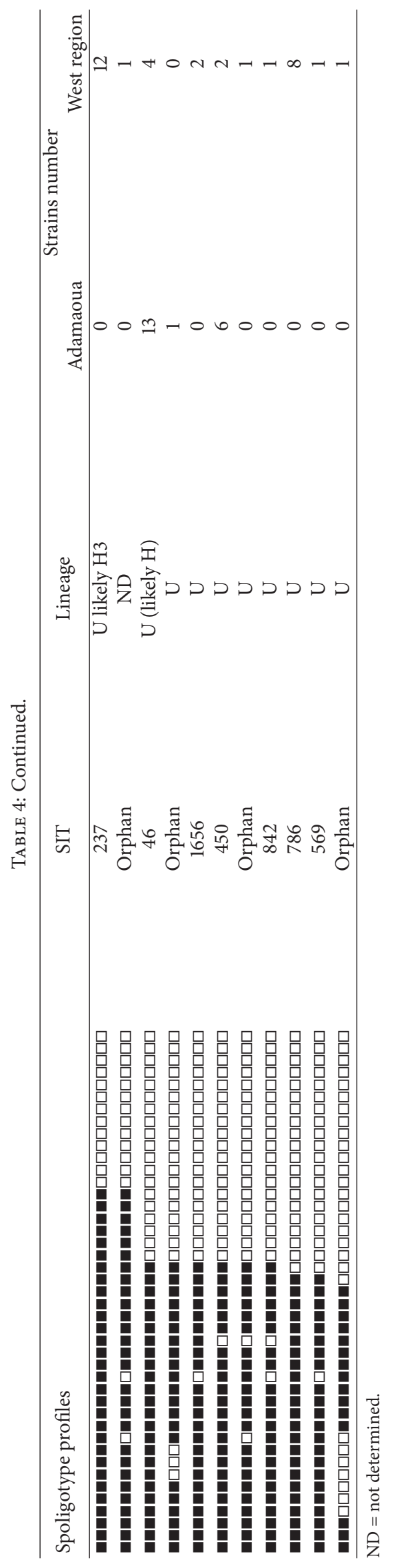


region while only three were isolated in Adamaoua (Table 4). Moreover 36 (55.4\%) spoligotypes among the sixty five found in Adamaoua were not found in West region. But these represent only $9.8 \%$ (43/437) of all isolated strains. Among these, 22 have never been described either in Cameroon or in the SITVIT_WEB databases.

3.6. Clonal Structure Analysis of the Most Prevalent Clade SIT61. The 34 (15\%) strains clonal structure of clade SIT61, randomly chosen, were analyzed with MIRU/VNTR using a modified standard 15-locus set. 19 haplotypes differing by more than two loci were identified (Table 5). This corresponds to allelic diversity of 0.93 . The discriminatory power between each locus varied between 0.00 and 0.67 . Seven loci were polymorphic: QUB-11b ( $\mathrm{H}=0.67)$, ETRA $(\mathrm{H}=0.59)$, MIRU40 $(\mathrm{H}=0.47)$, ETRC $(\mathrm{H}=0.34)$, MIRU $27(\mathrm{H}=0.34)$, QUB-26 $(\mathrm{H}=0.33)$, and MIRU $26(\mathrm{H}=0.16)$.

\section{Discussion}

It is now known that characterization of prevailing $M$. tuberculosis lineages and clones focusing on different geographical levels such as continents, countries, regions, or cities is important for locating the origin, evolution, and spreading dynamics of a particular M. tuberculosis clone [13]. Cameroon is a country of Golf of guinea with a high incidence of tuberculosis. It is particularly characterized by an expansion of a particular family of $M$. tuberculosis called "Cameroon family" and the decline of $M$. africanum which was previously widespread in the 1970s [14]. However all studies given this assertion had been only done in two of the ten regions that counts Cameroon. Systematic typing of MTBC population strains in another region of Cameroon was therefore needed in order to verify this assertion and for a better understanding of this damage disease's epidemiology in Cameroon.

We used for that purpose spoligotyping which is a rapid, simple, and reproducible molecular technic and which can be performed with nonpurified DNA [15-17]. It permits also the simultaneous distinction between MTBC strains at subspecies and lineage level. We also used MIRU/VNTR typing in order to analyze the clonally nature of the most prevalent clade SIT 61.

Ninety-seven percent of all TB cases reported in this study were caused by $M$. tuberculosis with the dominance of Cameroon family, largely represented by the lineage ST61. This result shows once again the prevalence of Cameroon family in Cameroon even in a rearing region as described in other studies $[2,3,10]$. The same result was obtained in neighboring Nigeria [6, 18], Chad [19], and other West African Countries, among which are Ghana [20] and Burkina Faso $[21,22]$.

The other M. tuberculosis families (T, Haarlem, and U) have also been identified in the Adamaoua region of Cameroon but with lesser proportion [3]. However some lineages that have never been described before in Cameroon were identified. This was X1 lineage which is ubiquitous in AngloSaxon countries and the U lineage ST523 clade which is scarce and only described in few countries like Nigeria and
USA. The occurrence of these families is probably due to the migration trade between Nigeria and Cameroon or to indirect zoonosis since this clade has been identified in strains causing tuberculosis in cattle in the same region [23]. None of the strains presenting the spoligotype of the worldwideexpanded epidemic Beijing family was identified. This result remains intriguing given the expansion capacity of this genotype and it identification in young girls in Kumba subdivision in Cameroon. We also identified only two spoligotypes as $M$. bovis; this was very intriguing because $M$. bovis is prevalent in cattle intended for human consumption in Cameroon [24], in Nigeria [6], and in Chad [25], two neighboring countries to Cameroon. Moreover Adamaoua region is the most rearing region of Cameroon. This low contribution of $M$. bovis and the dissemination of Cameroon family need to be further explored but we think as was the case of Beijing strain that the stain belonging to these species has different selective habits including virulence and pathogenesis or that systematical introduction of vaccination in new born baby in Cameroon may have selected some species or genotypes as Cameroon family. This situation could also be explained by differences in host reaction of African citizens. We also think that culinary habits of the Cameroon populations which eat well boiled and cooked meat could also explain this low contribution of $M$. bovis to human tuberculosis in Cameroon.

This result shows very low contribution of M. africanum to human pulmonary tuberculosis. But this contribution was lower than that obtained in other regions of Cameroon [2, 3]. This remains controversial since $M$. africanum was described as endemic in Cameroon in 1970s [14]. This result has been also observed in Guinea-Bissau a West African countries [26]. Conversely, other West African countries continue to report high proportions of $M$. africanum. Among them are Ghana with 20\% [20] and 23\% [27] of M. africanum and Nigeria with $13 \%$ [6] and $12.35 \%$ [18] of M. africanum.

The fact that $89 \%$ of $M$. africanum strains were not clustered argues in favor of reactivation of the old case than the ongoing transmission in Adamaoua. However, one clade of AFRI_2 lineage was clustered to four strains, suggesting that the strain of this clade might be actively implicated in TB transmission. But considering the few number of strains obtained, this assertion needs to be verified with other data and the use of other DNA markers different from the DR region. The same results were notified in Nigeria [18].

We also found in this study that $67 \%$ of $M$. africanum strains belonged to AFRI_2 lineage. This results were the same as those observed in West Cameroon where this lineage was also dominant and in neighboring Nigeria [18], but this was not the case in Guinea-Bissau where $99 \%$ of $M$. africanum strains responsible for active tuberculosis belonged to the AFRI_1 lineage.

The molecular investigation of Adamaoua's M. africanum strains did not show different results compared to those observed in West region of Cameroon after using the same techniques $[2,3]$. However, when looking at the global genetic structure, Cameroon family was significantly $(p=0.015)$ more represented in West region while $\mathrm{H} 1$ was significantly represented in Adamaoua $(p=0.033)$. 


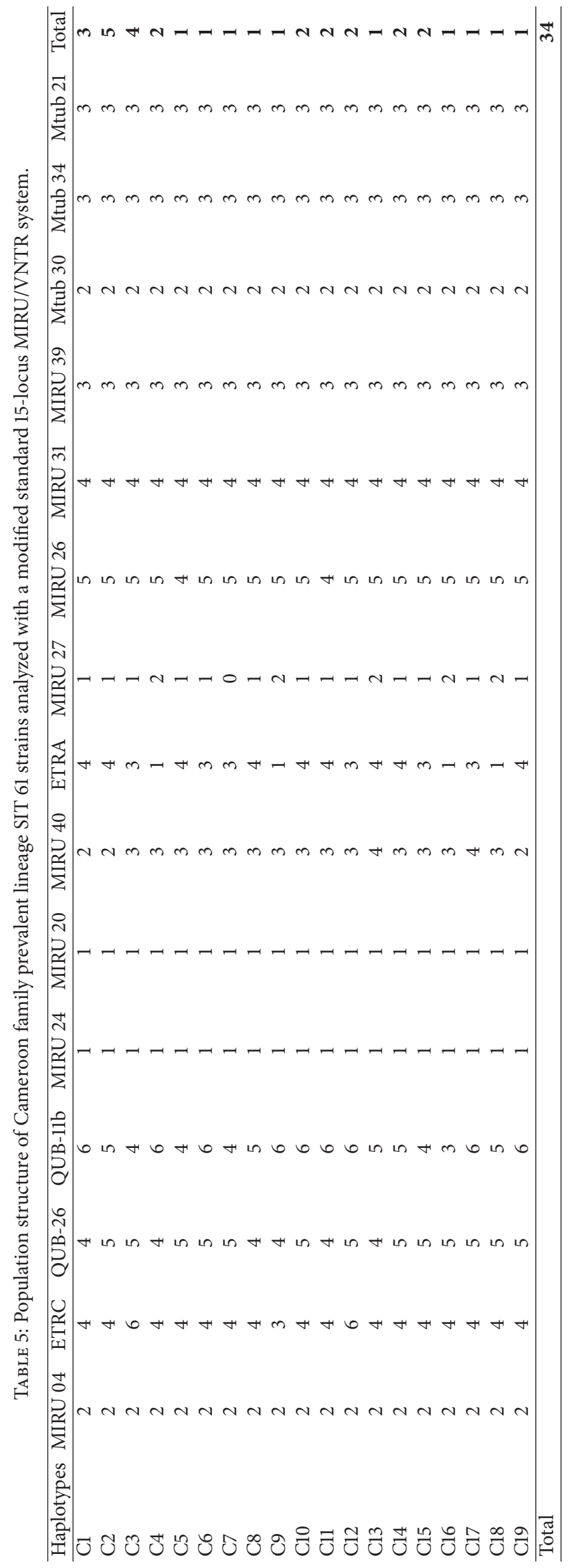


Clade ST61 in our study, based on spoligotyping results, seems to be associated with recent transmission since more than $80 \%(223 / 278)$ of strains of Cameroon family belong to this clade. The same result was found in neighboring Nigeria [18] and Burkina Faso [21]. But when analyzing molecular structure of the population of the strains belonging to this clade using independent markers (MIRU/VNTR), we obtained a high genetic diversity and a very high allelic diversity. This result suggested that this clade is not the true clone but that some of the strains were not implicated in the direct transmission of tuberculosis in Cameroon but it seems to be implicated in the reactivation of cases or in imported cases. However, some haplotypes such as C1, C2, and C3 were clustered with three, five, and four strains, respectively. This result could traduce the implication of strains of these haplotypes to different pockets of ongoing TB transmission in Adamaoua region.

The high diversity obtained in these lineages was very different to that obtained in the last study done in West region of Cameroon [10]. This difference might be due to the system of loci used in this first study in which the 12 standard MIRU/VNTR loci were used. In fact, four new polymorphic loci have been identified irrespective of what was obtained in the latest study.

Three other major families and lineages observed argue in favor of three different pockets of ongoing TB transmission; most of it is due to the ubiquitous T (T2 and T1) family, Haarlem family ( $\mathrm{H} 1$ and $\mathrm{H} 3$ lineages), and $\mathrm{U}(\mathrm{U}$ and $\mathrm{U}$ Likely $\mathrm{H}$ ) family phylogeographically specific for European and North American regions. We think that the presence of these families in Cameroon might be imported cases of the disease or strains that initially arrived through settlers but adapted to the local population. The same results have also been noticed in Nigeria [18].

The high prevalence of $M$. tuberculosis species and the very little prevalence of $M$. africanum and $M$. bovis in the Adamaoua lead us to evaluate the association between MTBC species or lineages and drug susceptibility. We found that only M. tuberculosis strains were responsible for all form of TB drug resistance observed in this study similarly to Ghana where $M$. tuberculosis species was also more likely resistant to any of the tested drugs compared to M. africanum [20]. The comparison of the drug resistance phenotypic proportions to different lineages did not permit us to find any statistical significant difference. Nevertheless only Cameroon family strains appeared to be associated with all phenotypic drug resistance observed, but in fewer proportions compared to other lineages like $\mathrm{T} 1$ when considering the phenotypic profile individually. This result also shows that Cameroon family presents the less proportion of MDR profiles compared to other M. tuberculosis lineages. This result can be intriguing considering the endemicity of this family in Cameroon but further studies analyzing the implication of this family to secondary drug resistant need to be done in order to better understand its endemicity in Cameroon.

\section{Conclusion}

Our study provides the first genetic structure of $M$. tuberculosis complex strain populations in the rearing region of Cameroun. Results show an expansion of the M. tuberculosis family endemic called Cameroon family in other regions and confirm a very low contribution of $M$. bovis and $M$. africanum to active pulmonary tuberculosis infection, even in the most rearing region of Cameroon.

\section{Competing Interests}

With the submission of this manuscript, the authors would like to undertake the responsibility that, for this submitted manuscript, they did not receive reimbursements, fees, funding, or salary from an organization that may in any way gain or lose financially from the publication of this manuscript, either now or in the future. They did not hold any stocks or shares in an organization that may in any way gain or lose financially from the publication of this manuscript, either now or in the future. Moreover they did not hold or were currently applying for any patents relating to the content of the manuscript or even received reimbursements, fees, funding, or salary from an organization that holds or had applied for patents relating to the content of the manuscript. To the best of their knowledge, they did not have any other financial and nonfinancial competing interests.

\section{Authors' Contributions}

Eyangoh Sara Irène, Um Boock Alphonse, Kuaban Christopher, Etoa François-Xavier, and Koro Koro Francioli conceived and designed the experiments. Koro Koro Francioli, Kaiyven Afi Leslie, and Noeske Juergen performed the experiments. Eyangoh Sara Irène and Gutierrez Cristina, Koro Koro Francioli, and Etoa François-Xavier analyzed the data. Koro Koro Francioli prepared the first draft of paper, and Gutierrez Cristina and Koro Koro Francioli designed the figures. All authors provided critical input.

\section{Acknowledgments}

This project received external grant funding from FAIRMED for sampling. The cost for genotyping was covered by the Centre Pasteur of Cameroon's internal resources. The authors acknowledge the support of the Centre Pasteur of Cameroon in the realization of this research. They also acknowledge $\mathrm{Mr}$. FOBASSO Romeo for English editing of this manuscript.

\section{References}

[1] A. Trébucq, "Rapport de l'Union International contre la tuberculose et les Maladies respiratoire," PNLT Rapport 2011, PNLT, 2011.

[2] S. N. Niobe-Eyangoh, C. Kuaban, P. Sorlin et al., "Genetic biodiversity of Mycobacterium tuberculosis complex strains from patients with pulmonary tuberculosis in Cameroon," Journal of Clinical Microbiology, vol. 41, no. 6, pp. 2547-2553, 2003. 
[3] F. K. Koro, Y. K. Simo, F. F. Piam et al., "Population dynamics of tuberculous bacilli in Cameroon as assessed by spoligotyping," Journal of Clinical Microbiology, vol. 51, no. 1, pp. 299-302, 2013.

[4] D. Van Soolingen, "Molecular epidemiology of tuberculosis and other mycobacterial infections: main methodologies and achievements," Journal of Internal Medicine, vol. 249, no. 1, pp. $1-26,2001$.

[5] S. N. Niobe-Eyangoh, C. Kuaban, P. Sorlin et al., "Genetic biodiversity of Mycobacterium tuberculosis complex strains from patients with pulmonary tuberculosis in Cameroon," Journal of Clinical Microbiology, vol. 41, no. 6, pp. 2547-2553, 2003.

[6] S. Cadmus, S. Palmer, M. Okker et al., "Molecular analysis of human and bovine tubercle bacilli from a local setting in Nigeria," Journal of Clinical Microbiology, vol. 44, no. 1, pp. 2934, 2006.

[7] UICTMR, Guide technique: Diagnostic de la tuberculose par examen microscopique direct des expectorations dans les pays à faibles revenus, Union Internationale Contre la Tuberculose et les Maladies Respiratoires (UICTMR), Paris, France, 2000.

[8] G. Cannetti, N. Rist, and J. Grosset, "Mesure de la sensibilité du bacille tuberculeux aux drogues antibacillaires par la méthodes des proportions," Revue de Tuberculose et de Pneumologie, vol. 27, pp. 217-272, 1963.

[9] J. Kamerbeek, L. Schouls, A. Kolk et al., "Simultaneous detection and strain differentiation of Mycobacterium tuberculosis for diagnosis and epidemiology," Journal of Clinical Microbiology, vol. 35, no. 4, pp. 907-914, 1997.

[10] S. N. Niobe-Eyangoh, C. Kuaban, P. Sorlin, J. Thonnon, V. Vincent, and M. C. Gutierrez, "Molecular characteristics of strains of the Cameroon family, the major group of Mycobacterium tuberculosis in a country with a high prevalence of tuberculosis," Journal of Clinical Microbiology, vol. 42, no. 11, pp. 50295035, 2004.

[11] P. R. Hunter and M. A. Gaston, "Numerical index of the discriminatory ability of typing systems: an application of Simpson's index of diversity," Journal of Clinical Microbiology, vol. 26, no. 11, pp. 2465-2466, 1988.

[12] K. Brudey, J. R. Driscoll, L. Rigouts et al., "Mycobacterium tuberculosis complex genetic diversity: Mining the fourth international spoligotyping database (SpolDB4) for classification, population genetics and epidemiology," BMC Microbiology, vol. 6, article 23, 2006.

[13] B. B. Asiimwe, S. Ghebremichael, G. Kallenius, T. Koivula, and M. L. Joloba, "Mycobacterium tuberculosis spoligotypes and drug susceptibility pattern of isolates from tuberculosis patients in peri-urban Kampala, Uganda," BMC Infectious Diseases, vol. 8, article 101, 2008.

[14] M. Huet, N. Rist, G. Boube, and D. Potier, "Etude bactériologique de la tuberculose au Cameroun," Revue de Tuberculose et de Pneumologie, vol. 35, pp. 413-426, 1971.

[15] M. Goyal, S. Lawn, B. Afful, J. W. Acheampong, G. Griffin, and R. Shaw, "Spoligotyping in molecular epidemiology of tuberculosis in Ghana," Journal of Infection, vol. 38, no. 3, pp. 171-175, 1999.

[16] L. Qian, J. D. A. Van Embden, A. G. M. Van Der Zanden, E. F. Weltevreden, H. Duanmu, and J. T. Douglas, "Retrospective analysis of the Beijing family of Mycobacterium tuberculosis in preserved lung tissues," Journal of Clinical Microbiology, vol. 37, no. 2, pp. 471-474, 1999.

[17] S. Roring, M. S. Hughes, R. A. Skuce, and S. D. Neill, "Simultaneous detection and strain differentiation of Mycobacterium bovis directly from bovine tissue specimens by spoligotyping," Veterinary Microbiology, vol. 74, no. 3, pp. 227-236, 2000.

[18] B. P. Thumamo, A. E. Asuquo, L. N. Abia-Bassey et al., "Molecular epidemiology and genetic diversity of Mycobacterium tuberculosis complex in the Cross River State, Nigeria," Infection, Genetics and Evolution, vol. 12, no. 4, pp. 671-677, 2012.

[19] C. Diguimbaye, La tuberculose humaine et animale au Tchad: contribution à la mise en évidence et caractérisation des agents causaux et leur implication en santé publique [Ph.D. thesis], Faculty of Science, University of Basel, 2004.

[20] D. Yeboah-Manu, A. Asante-Poku, T. Bodmer et al., "Genotypic diversity and drug susceptibility patterns among $\mathrm{M}$. tuberculosis complex isolates from South-Western Ghana," PLoS ONE, vol. 6, no. 7, Article ID e21906, 2011.

[21] S. Godreuil, G. Torrea, D. Terru et al., "First molecular epidemiology study of Mycobacterium tuberculosis in Burkina Faso," Journal of Clinical Microbiology, vol. 45, no. 3, pp. 921-927, 2007.

[22] M. K. Gomgnimbou, G. Refrégier, S. P. Diagbouga et al., "Spoligotyping of Mycobacterium africanum, Burkina Faso," Emerging Infectious Diseases, vol. 18, no. 1, pp. 117-119, 2012.

[23] F. Koro Koro, A. F. Ngatchou, J. L. Portal, C. Gutierrez, F.-X. Etoa, and S. I. Eyangoh, "The genetic population structure of Mycobacterium bovis strains isolated from cattle slaughtered at the Yaoundé and Douala abattoirs in Cameroon," Revue Scientifique et Technique, vol. 34, pp. 1001-1010, 2015.

[24] F. Koro, Bouba, E. Foko, A. F. Ngatchou, S. Eyangoh, and F.-X. Etoa, "First insight into the current prevalence of bovine tuberculosis in cattle slaughtered in Cameroon: the case of main abattoirs of Yaoundé and Douala," British Microbiology Research Journal, vol. 3, no. 3, pp. 272-279, 2013.

[25] C. Diguimbaye, M. Hilty, R. Ngandolo et al., "Molecular characterization and drug resistance testing of Mycobacterium tuberculosis isolates from Chad," Journal of Clinical Microbiology, vol. 44, no. 4, pp. 1575-1577, 2006.

[26] R. Groenheit, S. Ghebremichael, J. Svensson et al., "The GuineaBissau family of Mycobacterium tuberculosis complex revisited," PLoS ONE, vol. 6, no. 4, Article ID e18601, 2011.

[27] K. Addo, K. Owusu-Darko, D. Yeboah-Manu et al., "Mycobacterial species causing pulmonary tuberculosis at the korle bu teaching hospital, Accra, Ghana," Ghana Medical Journal, vol. 41, no. 2, pp. 52-57, 2007. 


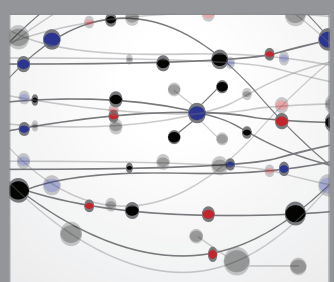

The Scientific World Journal
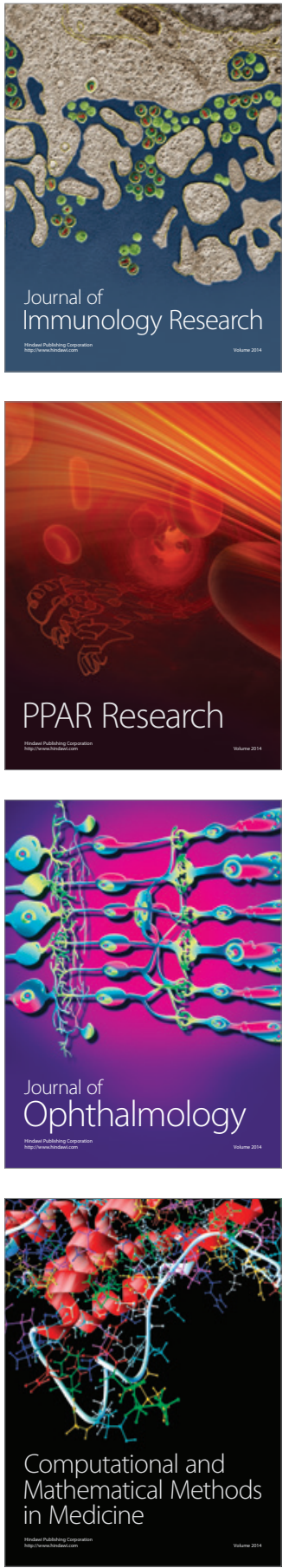

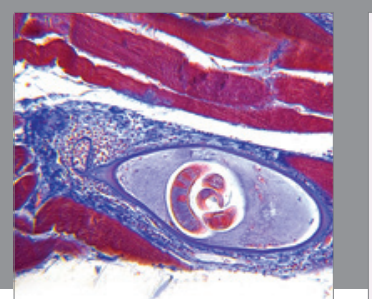

Gastroenterology Research and Practice

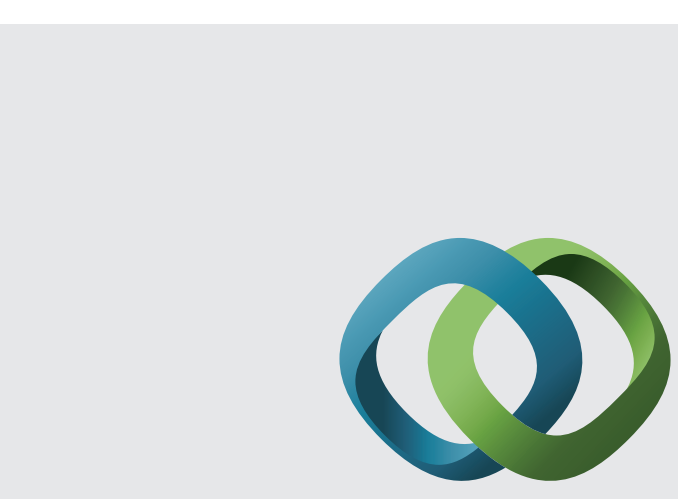

\section{Hindawi}

Submit your manuscripts at

http://www.hindawi.com
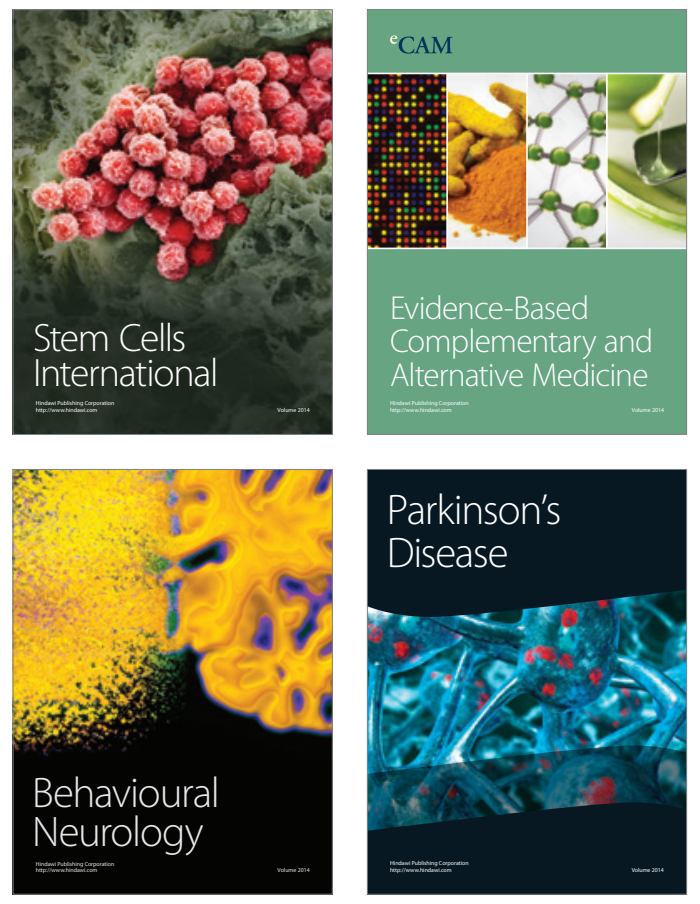
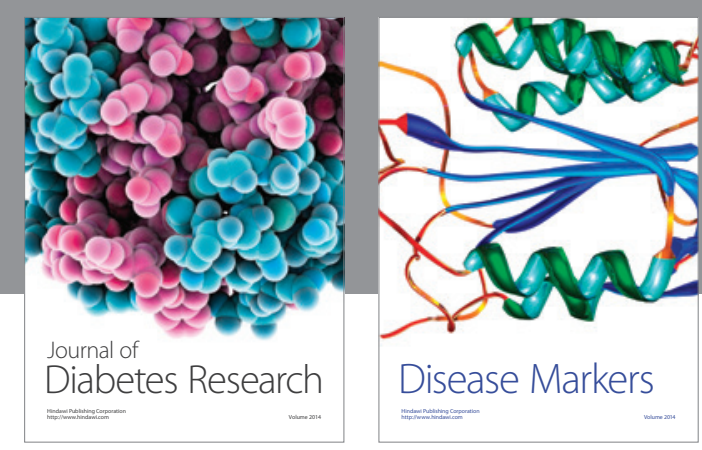

Disease Markers
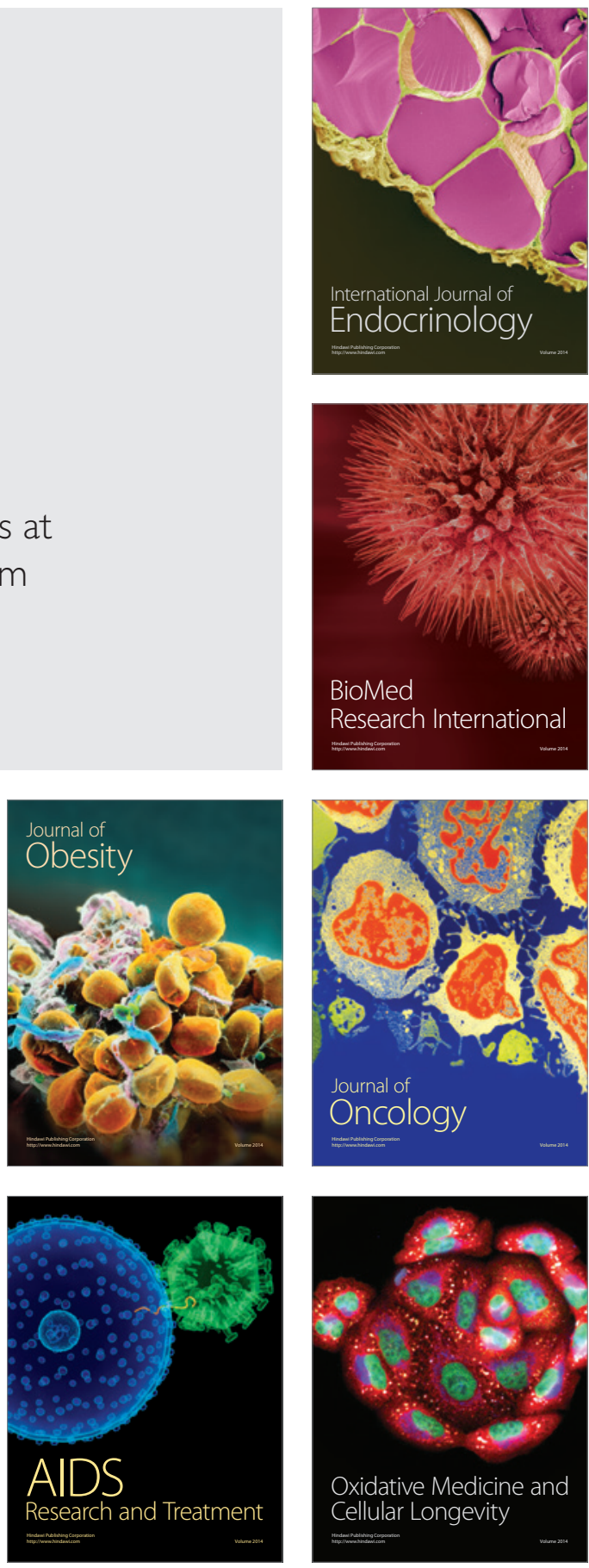